\title{
Doomscrolling During COVID-19: The Negative Association Between Daily Social and Traditional Media Consumption and Mental Health Symptoms During the COVID-19 Pandemic
}

*For correspondence:

Matthew.Price@uvm.edu (MP)

Present address: 2 Colchester Ave, Burlington VT 05405

\begin{abstract}
Matthew Price $^{1 *}$, Alison C. Legrand ${ }^{1}$, Zoe M. F. Brier ${ }^{1}$, Katherine van Stolk-Cooke ${ }^{1}$, Kelly Peck ${ }^{2,3}$, Peter Sheridan Dodds ${ }^{4}$, Christopher M. Danforth ${ }^{5}$, Zachary Adams ${ }^{6}$

${ }^{1}$ Center for Research on Emotion, Stress, and Technology, Department of Psychological Science, University of Vermont; ${ }^{2}$ Vermont Center on Behavior and Health and University of Vermont; ${ }^{3}$ Departments of Psychiatry, University of Vermont; ${ }^{4}$ Department of Computer Science, Vermont Complex Systems Center, University of Vermont; ${ }^{5}$ Department of Mathematics Statistics, Vermont Complex Systems Center, University of Vermont; ${ }^{6}$ Adolescent Behavioral Health Research Program, Department of Psychiatry, Indiana University School of Medicine, Indianapolis, IN
\end{abstract}

\section{Disclosure}

(C) 2021, American Psychological Association. This paper is not the copy of record and may not exactly replicate the final, authoritative version of the article. Please do not copy or cite without authors' permission. The final article will be available, upon publication, via its DOI: 10.1037/tra0001202

\section{Abstract}

Objective: Consumption of traditional and social media markedly increased at the start of the COVID-19 pandemic as new information about the virus and safety guidelines evolved. Much of the information concerned restrictions on daily living activities and the risk posed by the virus. The term "doomscrolling" was used to describe the phenomenon of elevated negative affect after viewing pandemic-related media. The magnitude and duration of this effect, however, is unclear. 
Furthermore, the effect of doomscrolling likely varies based on prior vulnerabilities for psychopathology such as a history of childhood maltreatment. It was hypothesized that social and traditional media exposure were related to an increase in depression and PTSD and that this increase was moderated by childhood maltreatment severity. Method: Participants completed a baseline assessment for psychopathology and 30 days of daily assessments of depression, PTSD, and pandemic-related media use. Results: Using multilevel modeling, social media exposure was associated with increased depression and PTSD. This association was stronger for those with more severe maltreatment histories. Furthermore, those with more severe baseline psychopathology used more social media during this period. These relations were not observed for traditional media sources. Conclusions: These results suggest that regular viewing of pandemic-related social media is associated with increases in psychopathology for those with existing vulnerabilities. Those with such vulnerabilities should adopt strategies to limit social media consumption.

\section{Introduction}

The COVID-19 pandemic altered the global mental health landscape for young adults (Galea et al., 2020). Preliminary reports demonstrated that changes to daily living caused by the pandemic collectively increased psychopathology including depression and anxiety (Fullana et al., 2020; Wang et al., 2020). These changes included the need for social distancing, wearing of face coverings, and closures of businesses and schools. Information about these changes were largely disseminated through traditional news sources and social media. The near ubiquitous reach of modern day media and the increased use of such media by younger individuals (Auxier Anderson, 2021) has made understanding its impact on mental health during the pandemic critical (Holmes et al., 2020). The extent to which repeated exposure to pandemic-related media impacts mental health is unclear, especially for those with pre-pandemic risk factors for psychopathology such as childhood maltreatment. Determining the direction and strength of this association is critical to understanding the scale of the pandemic's effect on mental health among those with existing vulnerabilities.

The Social Amplification of Risk framework offers a model to explain how repeated exposure to such media may increase psychopathology (Kasperson et al., 1988). This framework suggests that a public health risk can be amplified in the transmission of new information via messaging valence and frequency. Information delivered via the internet, including social media and news alerts, is often negatively valenced such that it includes risk-amplifying language (Sell et al., 2017). Indeed, a review of messaging related to COVID-19 specifically highlighted that many messages use negative and fear-based language (Heffner et al., 2021). Mobile devices allow frequent access to such media as well. The negative valence coupled with regular access and exposure to such content could serve to magnify risk perceptions. Prior work has shown the utility of this framework in understanding the response to a public health emergency (Ng et al., 2018). Those exposed to negatively valenced media about haze related to air pollution reported increased perceived risk and negative affect.

As evidence of the effect of risk amplification, the term "doomscrolling" was coined during the pandemic and gained widespread use (Figure 1; Alshaabi et al., 2021). Doomscrolling describes the surge in negative affect associated with repeated exposure to pandemic-related stories. The magnitude and duration of this effect, however, remains unclear. It is also unclear if the source of the material is associated with the magnitude of the effect. Social media may differentially impact 
psychopathology than traditional media given differences in personal connection to the authors and possible differences in overall message valence. Furthermore, if it unclear if prior vulnerabilities for psychopathology, such as a history of childhood maltreatment or a prior mental health diagnosis, may exacerbate the effect of doomscrolling. Understanding these relations are important to provide clinical guidance to those who may experience a rise in psychopathology symptoms during the pandemic.

Figure 1. Figure 1. Frequency of the use of the of the term "doomscrolling" on Twitter from January 27th, 2020 through January 29th, 2021. Y-axis is logarithmic scale. Demonstrates the appearance and frequent use of the term on social media.

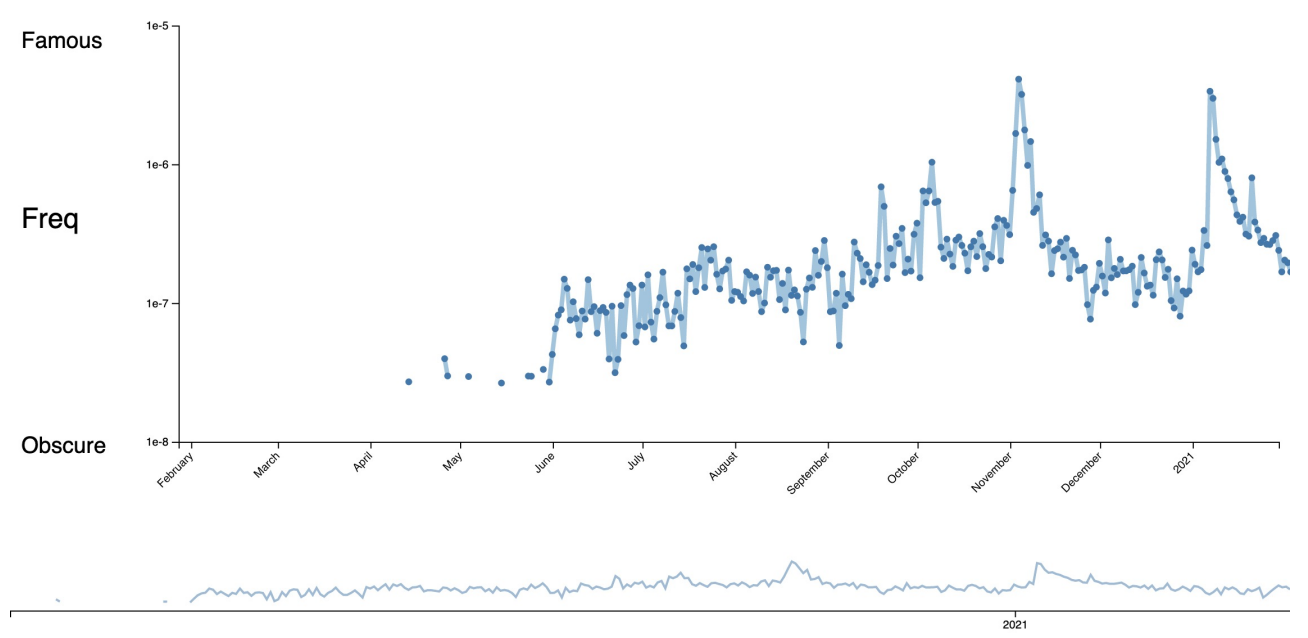

Young adults are a population of considerable interest in determining the impact of exposure to pandemic-related media. Those within early adulthood saw a substantial increase in the severity and frequency of psychopathology during the COVID-19 pandemic (Gonzales et al., 2020; Li et al., 2021). This age group is also more likely to use mobile devices and social media on a regular basis, including in a problematic manner (Auxier Anderson, 2021; Contractor et al., 2019). Indeed, $84 \%$ of young adults use social media daily. Thus, it is necessary to determine if regular exposure to COVID related media is associated with changes in psychopathology in this population.

There is limited literature on the relation between repeated exposure to media and mental health during an ongoing stressor such as the COVID-19 pandemic in young adults. Much of the prior work on media exposure and psychopathology has come from events of mass trauma. Regular exposure to media coverage of the Boston Marathon bombing was associated with increased acute stress symptoms (Holman et al., 2014). However, mass traumas are often acute events, whereas the COVID-19 pandemic was an ongoing, multi-year-long stressor. The persistent nature of the pandemic required individuals to remain informed via media about ongoing changes to daily living whereas media after acute events largely reported on prior events. This difference may alter the psychological impact of regularly viewing media as the information is pertinent to an ongoing event.

Moreover, little is known about the longitudinal impact of sustained media exposure on mental health during an ongoing disaster in young adults. Although much research is ongoing, several studies have demonstrated a positive relation between media exposure related to COVID19 and symptoms of depression and anxiety in samples obtained in China and Spain (Fullana et al., 2020; Gao et al., 2020; Wang et al., 2020). These studies were cross sectional, used retrospective reports of media use and focused largely on middle aged individuals. Although this provides an overview of the association between an ongoing public health stressor and media exposure, it 
does not provide information as to how daily exposure influences fluctuations in mental health symptoms. With mobile devices, individuals have access to news media on demand with a near limitless supply of information. Longitudinal methods that evaluate daily exposure, such as daily diary studies, are needed to examine this relation. Such studies allow for an examination of the association between daily media exposure and daily mental health. Given the ongoing nature of the pandemic, this approach is needed to determine if changes in psychopathology related to media exposure were temporary, such that they faded quickly, or persistent, such that cumulative exposure gradually increased severity. Understanding this relation is particularly important for young adults given the range of disruptions to their lives that included the closing of institutions of higher education, closing of places of work, and loss of social interactions with others.

Those with histories of childhood maltreatment may be more susceptible to the negative impacts of repeated media exposure. A prior study suggested that young adults with histories of childhood maltreatment were more likely to engage in problematic internet use broadly (Yates et al., 2012). This type of internet use is defined as compulsive, used for comfort seeking, and has a negative impact on social, academic, and occupational related functions. Another study with a similarly aged sample reported a relation between more problematic social media use specifically and childhood maltreatment severity (Worsley et al., 2018). The increased social media use among those with such histories was proposed as a coping mechanism. That is, those with histories of childhood maltreatment may have fewer coping strategies available in times of distress and thus turn to social media. Social media, however, may exacerbate their symptoms given the overall negative valence of much of the available content. Indeed, adults with PTSD have been shown to engage in more problematic mobile phone use (Contractor et al., 2019). A limitation of this work is the reliance on retrospective self-reports of social media use as opposed to longitudinal assessments of actual media use. Thus, it remains unclear as to the extent that actual social media use during the pandemic was associated with changes in psychopathology among young adults with histories of childhood maltreatment.

The present study evaluated the association between daily exposure to pandemic-related media and symptom severity for depression and posttraumatic stress disorder (PTSD) in young adults. The present study used a daily diary methodology to measure this relation over 30 days. Furthermore, comparisons were made between social media and traditional media given the potential for social media to have a stronger negative valence than traditional media. It was hypothesized that daily exposure to pandemic-related news media and social media would be associated with elevated symptom severity for each disorder. It was also hypothesized that the magnitude of the increase in symptom severity after viewing media would be moderated by the severity of childhood maltreatment history. To determine the extent that those with elevated psychopathology may be more prone to turn to social media, the relation between baseline psychopathology and overall social and traditional media use during the first month of the pandemic was examined. Finally, the cumulative effect of media consumption on PTSD and depression symptoms during the month-long assessment period was also evaluated.

\section{Methods}

\section{Participants}

Participants were 61 young adults (Myears $=18.89$, SDyears $=0.90$ ) who participated in a larger study on the impact of childhood maltreatment on neurological mechanisms of psychopathology (Table 1). Participants were recruited for the parent study as those with elevated histories of childhood maltreatment $(n=41)$ and those with minimal exposure to childhood maltreatment $(n=20)$. Inclusion criteria required a score on the Adverse Childhood Experiences Scale 


\begin{tabular}{lcc}
\hline & $M$ & $S D$ \\
Age & 18.89 & 0.90 \\
Baseline Pandemic Depression (PHQ-9) & 9.45 & 6.45 \\
Baseline Pandemic PTSD (PCL-5) & 26.26 & 19.02 \\
Childhood Trauma Questionnaire Total Score & 44.32 & 17.74 \\
\hline & $N$ & $\%$ \\
Sex (Female) & 53 & 86.7 \\
Transgender & 5 & 8.2 \\
hace & & \\
$\quad$ White & 55 & 90.2 \\
$\quad$ African American & 2 & 3.3 \\
$\quad$ Asian American & 1 & 1.6 \\
$\quad$ Biracial & 3 & 4.9 \\
& & \\
Annual Household Income & & \\
$\quad<\$ 30,000$ & 21 & 34.4 \\
$\quad \$ 30,000-\$ 50,000$ & 12 & 19.7 \\
$>\$ 50,000$ & 24 & 39.3 \\
History of Major Depressive Episodes & & \\
History of PTSD & 16 & 26.2 \\
\hline
\end{tabular}

Table 1. Descriptive information about the sample $(N=61)$.

(ACEs) greater than 3 and experiencing childhood maltreatment prior to age 18. Childhood maltreatment was defined as an experience that would have warranted disclosure to child protective services. The childhood maltreatment status was verified by a trained research assistant during a diagnostic interview. For those in the comparison group, inclusion criteria required a score on the ACES $<3$ and lack of any identifiable childhood maltreatment during the diagnostic interview. All participants were aged 18-20 years. Exclusion criteria included having a psychotropic medication change within the past two months.

Measures

MINI International Neuropsychiatric Interview for the DSM 5 (MINI: Sheehan et al., 1998). The MINI is a semi-structured interview that assessed the presence of mental health disorders according to the DSM 5. The MINI was administered prior to the start of the pandemic as part of the parent study. For the present study, the MINI was used to assess a history of major depressive disorder (MDD). Interviews were administered by trained research assistants. A licensed clinical psychologist reviewed $20 \%$ of interviews to determine diagnostic accuracy. Agreement was found to be 100

Clinician Administered PTSD Scale (CAPS; Weathers et al., 2018). The CAPS is the gold standard clinical interview for the assessment of PTSD in adult samples. The CAPS was administered prior to the start of the pandemic as part of the parent study to determine the presence of a PTSD diagnosis to all participants. Interviews were administered by trained research assistants. A licensed clinical psychologist examined concordance rates for $20 \%$ of interviews. Agreement was found to be 100

Childhood Trauma Questionnaire (CTQ: Bernstein et al., 1997). The CTQ is a 28-item self-report scale that assesses five categories of negative childhood experiences: emotional ne- 
glect, emotional abuse, physical neglect, physical abuse, and sexual abuse, and has excellent psychometric properties. A total scale with scores ranging from 5-125 is obtained with higher scores indicating greater abuse. Internal consistency for the CTQ was excellent in the current sample, $\alpha^{\prime} s=.91$.

Patient Health Questionnaire - 9 (PHQ-9: Kroenke et al., 2001). The PHQ-9 is a validated measure of depressive symptoms. Symptoms of depression are rated on a 0-27 scale with higher scores indicating more severe depression. Internal consistency for the PHQ-9 was excellent, $\alpha^{\prime} s>.91$.

PTSD Checklist for DSM-5 (PCL-5; Bovin et al., 2016). The PCL-5 is a 20-item self-report measure of PTSD symptoms. Ratings are made on a 0-80 scale, with higher scores indicating more severe PTSD symptoms. Internal consistency for the PCL- was excellent, $\alpha^{\prime} s>.95$.

Media Exposure. Media exposure was measured with a multi-choice question - "How did you get any news about the coronavirus today?" The choices were: Social media (Instagram, Twitter, Facebook, etc.), television, radio, podcast, newspaper or newspaper app or newspaper website, phone news alert, and "I didn't get any news today about the coronavirus". Participants were allowed to select multiple modalities for a given day. For the present study, this question was dichotomized to no exposure and exposure. Cumulative exposure was calculated by using the cumulative sum of the days of media exposure for the 30-day assessment period.

\section{Procedure}

Data collection for the present study occurred in two phases. The first phase was conducted as part of a larger study examining the association between childhood maltreatment and psychopathology in young adults. Participants were recruited from the community via flyers, online advertisements, and partnerships with local advocacy agencies that work with youth with maltreatment histories. Recruitment occurred from July 18th, 2018 to February 13th, 2020. A total of 74 participants completed study procedures. Participants completed the MINI, CAPS, and CTQ as part of a larger assessment battery for this phase. The first cases of COVID-19 in Vermont, where the study was conducted, was recorded on March 7th, 2020. Businesses and schools closed, and stay-at-home orders were issued between March 10th and March 24th, 2020. Enrollment began on April 4th, 2020, all participants were contacted to complete a measure battery that included the baseline PHQ-9 and PCL-5. Of those contacted, 62 (83.8\%) completed the assessment. Of those who did not participate, 2 (2.7\%) declined and 10 (13.5\%) did not respond. There were no significant differences on pre-pandemic measures between those who responded and those who did not.

Participants were then invited to participate in the second phase; a daily diary study in which they completed a daily assessment that included a PHQ-9, PCL-5, and the media exposure question for 30 days. One participant completed only 1 assessment and was removed from all analyses, resulting in an analyzed sample of 61 . Notifications to complete the daily survey were sent via automated text using the Survey Signal platform (Chicago, IL). Notifications were sent randomly between 7:00PM and 8:30PM with a reminder sent 2 hours later if responses were not provided within that timeframe. The notification text included a link to an online survey that was administered via Qualtrics. In total, participants had until 7AM the following day to complete the survey.

Data Analytic Plan

The hypotheses about daily media exposure were examined within a multilevel modeling framework with separate models for each outcome (depression, PTSD). The outcomes of interest 
were daily symptom scores. The fixed effects of interest were the slope reflecting linear change in outcomes for the assessment period, the time-varying predictor of exposure to COVID-19-related social and news media, and the interactions between media exposure and childhood maltreatment. Additional variables included as covariates were pre-COVID diagnoses of MDD and PTSD, and gender. Time was scaled in days with 0 fixed to the first day of the assessment period. Random effects were evaluated by model comparisons between those that did and did not contain random intercepts and slopes.

The relation between baseline psychopathology and cumulative media exposure was assessed with a linear regression model. Predictors were baseline depression and PTSD symptoms as well as history of childhood maltreatment. The outcome was the number of days in which social media and traditional media were viewed. Separate models were used for each outcome. Covariates included pre-pandemic diagnoses, childhood maltreatment severity, and gender.

To assess hypotheses about the effect of cumulative exposure on psychopathology, a linear regression model was used. Outcomes were severity of depression and PTSD at 1-month follow-up while controlling for symptom severity at the start of the pandemic. Predictors of interest included the sum of days of exposure to social media and sum of exposure to other news media. Covariates included baseline levels of psychopathology at the start of the pandemic, a prepandemic diagnosis of major depressive episodes or PTSD, gender, and childhood trauma severity. All study procedures were approved by the University of Vermont IRB.

Missing data were handled using full information maximum likelihood estimation, which has shown to provide accurate parameter estimates using multilevel models with this degree of missingness (Larsen, 2011; Schlomer et al., 2010). Data for the current analysis were aligned to a specific date rather than an individual's period within the study. Measurements were anchored to date to account to ensure that all measurements were associated with similar news cycles. Participants were enrolled on different dates, however, and so the missing data rate was $19.6 \%$. The proportion of missing data increased over the 30 -day period. A sensitivity analysis was conducted to determine the impact of the larger portion of missing data towards the end of the 30-day period. The analyses were conducted again using periods of 10,15, 20, 25 days to determine if the results changed by using shorter periods with more complete data. Across all periods, the pattern of results was identical. Finally, missingness was unrelated to a history of PTSD $(t=-0.91,=.366)$, prior history of depression $(t=-1.22, p=.233)$, PTSD symptoms at the start of the pandemic $(b<.01, p$ $=.424)$, depression symptoms at the start of the pandemic $(\mathrm{b}<.01, \mathrm{p}=.184)$, PTSD symptoms at the conclusion of the 30 -day period $(b<.01, p=.681)$, or depression symptoms at the conclusion of the 30 day period $(b<.01, p=.728)$. Therefore, results from the 30 -day period are presented."

\section{Results}

Models with random slopes had better fit for PTSD and depression and were used in subsequent analyses (PTSD: $\chi^{2}(2)=47.60, p<.001$; Depression: $\chi^{2}(2)=30.37, p<.001$ ). An initial model suggested that depression $(b=-0.05, p<.001)$ and PTSD $(b=-0.08, p=.012)$ symptoms significantly decreased during the 30 -day assessment period. Childhood maltreatment was associated with depression $(b=0.16, p<.001)$ and PTSD $(b=0.31, p<.001)$ symptom severity. A prior diagnosis of PTSD was associated with elevated PTSD symptoms $(b=9.90, p=.002)$. However, $a$ prior diagnosis of major depressive episodes was unrelated to current symptoms. Gender was not associated with either outcome. COVID-19 related social media was accessed on $44.6 \%$ of the assessments whereas news media was accessed for $34.3 \%$ of the assessments. Newspapers were the most accessed traditional media source (23.7\%), followed by television (16.4\%), Radio (3.7\%), and Podcasts (2.2\%). The extent that the association between viewing social media and traditional 
media and psychopathology varied over time was then explored. Interaction terms between time and each media type were not significant for PTSD (Social Media: $b=-0.12, p=.101$; Traditional Media: $b=0.12, p=.102$ ) and depression (Social Media: $b=-0.02, p=.474$; Traditional Media: $b=$ $0.01, p=.682$ ).

Multilevel models examined the role of media exposure and childhood maltreatment on psychopathology (Table 2). For depression, there was a significant interaction between childhood maltreatment histories and social media exposure $(b=0.03, p=.044)$. Probing this interaction at the different levels of social media exposure indicated that depression was elevated on days when social media was viewed and that this increase was greater for those with more severe childhood maltreatment histories (Figure 1). The mean effect size of exposure to social media on depression was $d=0.44,95 \% \mathrm{Cl}: 0.31$ to 0.57 .

Figure 2. Model implied interaction of the association between depression (PHQ-9) and social media access at different levels of CTQ scores (childhood maltreatment). The left figure corresponds to CTQ scores 1 standard deviation below the mean, the central figure corresponds to CTQ scores at the mean, and the right panel corresponds to CTQ scores 1 standard deviation above the mean.

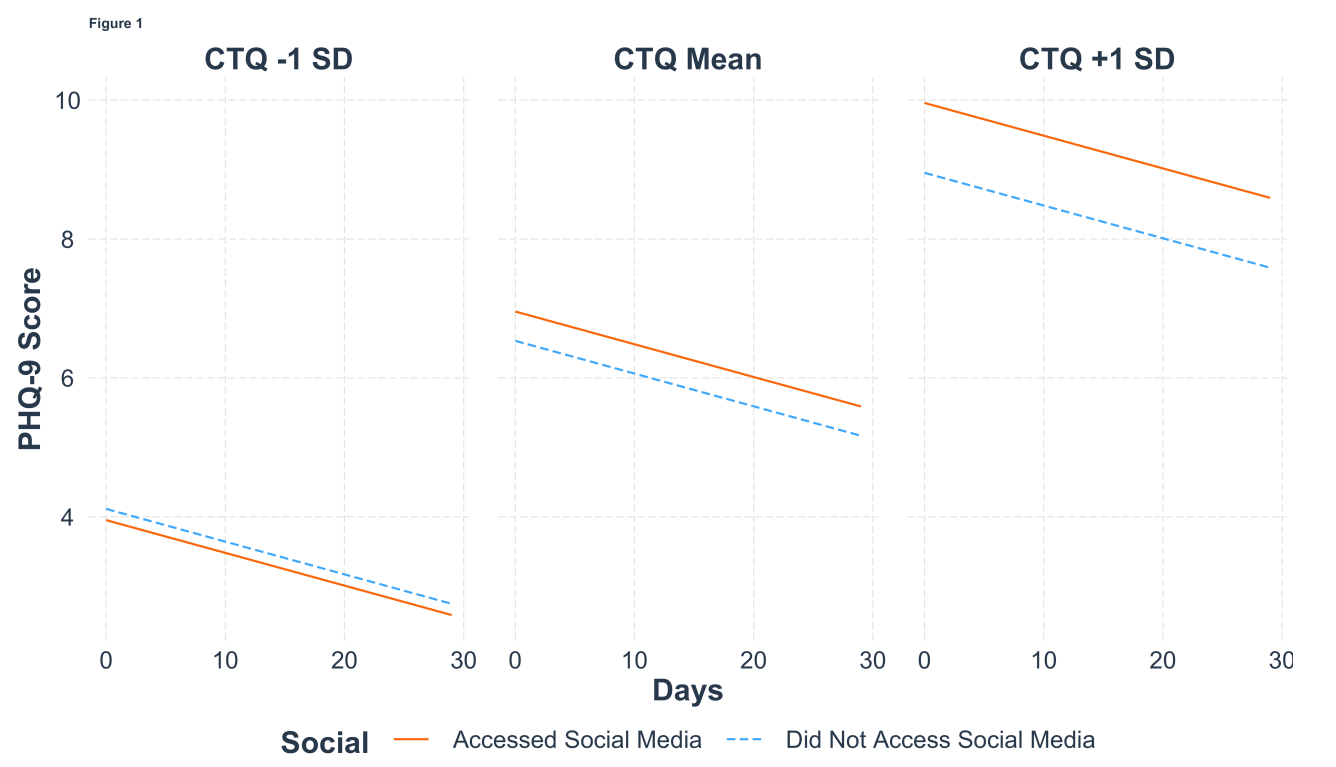

For PTSD, there was also a significant interaction between childhood maltreatment histories and social media access $(b=0.11, p=.002)$. Probing this interaction at the levels of social media access revealed a similar pattern of findings to that of depression. That is, social media was associated with elevated PTSD symptoms and the magnitude of this increase was associated with the severity of childhood maltreatment history (Figure 3). The mean effect size of accessing social media on PTSD was $d=0.36,95 \% \mathrm{Cl}: 0.21$ to 0.52 . There were no significant main effects or an interaction between traditional media and childhood maltreatment history for depression or PTSD.

The association between baseline psychopathology and number of days of social media and traditional media exposure was then examined. Baseline PTSD $(b=0.17, \mathrm{SE}=0.07, \mathrm{p}=.012)$ and depression $(b=0.58, \mathrm{SE}=0.17, \mathrm{p}=.001)$ were positively associated with social media use during the start of the pandemic (Table 3). There were no main effects for childhood maltreatment history or significant interactions with baseline psychopathology. There were no significant associations between psychopathology and number of days of traditional media exposure. Finally, the influence of cumulative exposure on psychopathology was assessed. There were no significant effects of cumulative exposure to social media or traditional media on changes in PTSD or depression during 
Figure 3. Model implied interaction of the association between PTSD (PCL-5) and social media access at different levels of CTQ scores (childhood maltreatment). The left figure corresponds to CTQ scores 1 standard deviation below the mean, the central figure corresponds to CTQ scores at the mean, and the right panel corresponds to CTQ scores 1 standard deviation above the mean.

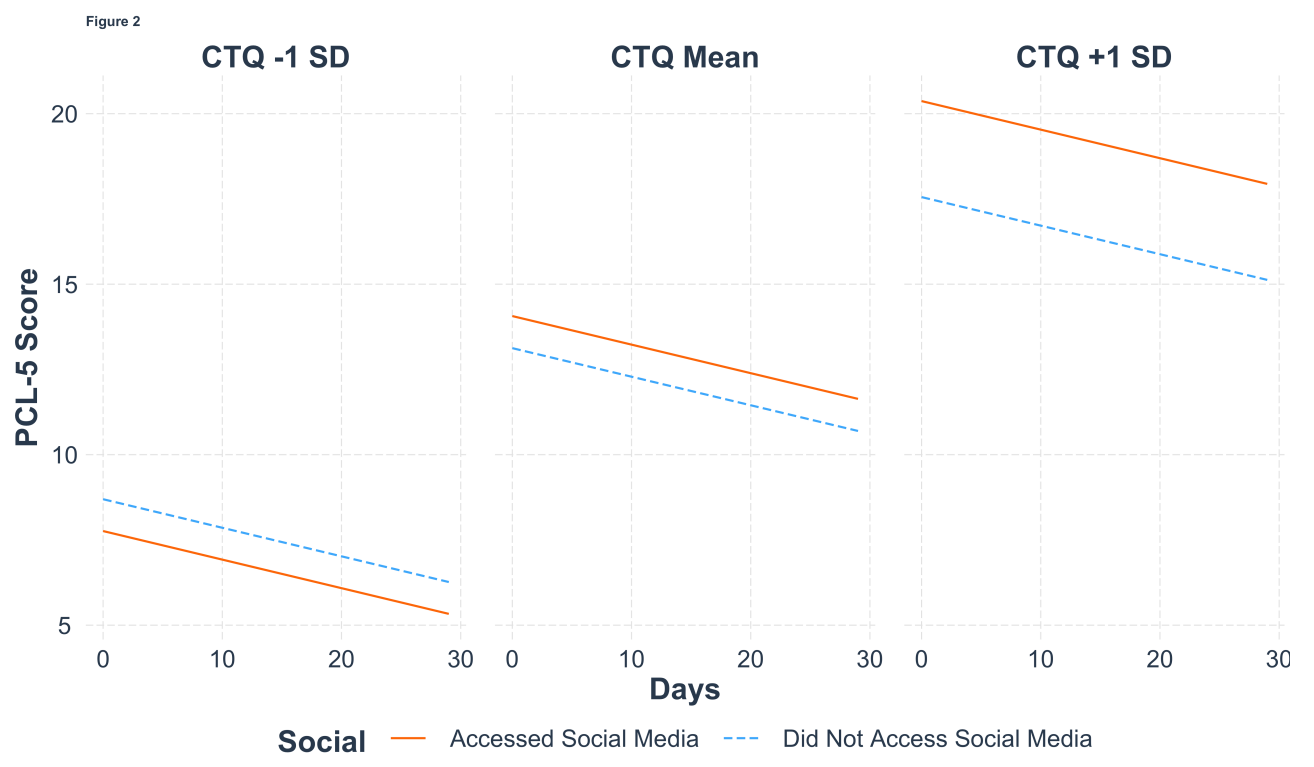

this month. Furthermore, interactions between childhood maltreatment history and cumulative exposure to social media were not significant.

\section{Discussion}

The results of the present study showed an association between daily exposure to pandemicrelated social media and elevated depression and PTSD symptoms in young adults during the initial months of the COVID-19 pandemic in the United States. This relation was moderated by a history of childhood maltreatment such that those with a more severe history experienced a greater increase. This set of associations was not found for exposure to traditional media during the same period. Furthermore, social media was viewed more frequently than traditional media. Taken together, these findings suggest that exposure to social media during the pandemic may have exacerbated mental health conditions for those with prior vulnerabilities (Holmes et al., 2020).

The association between social media exposure and psychopathology among those with childhood maltreatment histories is likely bidirectional. Those with elevated depression and PTSD at the start of the pandemic viewed social media more frequently. Prior work on the use of social media among young adults with maltreatment histories posited that overuse of social media may be a coping strategy to alleviate distress (Worsley et al., 2018). Those with histories of childhood maltreatment have been shown to more often rely on maladaptive coping strategies (Perlman et al., 2016). Thus, those with elevated psychopathology when the pandemic began may have turned to social media more regularly for information about the pandemic to reduce symptoms of psychopathology.

The social amplification of risk framework also suggests the opposite relation - that viewing social media may exacerbate psychopathology. The study was conducted early in the pandemic when restrictions were being put into place on travel, businesses and schools were in the process of closing, and social distancing measures were put into effect. It is likely that the social media 


\section{Depression (PHQ-9)}

\begin{tabular}{lccc} 
Variable & $b$ & SE & $p$ \\
\hline Time & -0.05 & 0.01 & $<.001$ \\
Social Media Exposure & -0.83 & 0.66 & .206 \\
Traditional News Media Exposure & 0.27 & 0.62 & .660 \\
CTQ & 0.05 & 0.03 & .069 \\
Baseline Depression (PHQ-9) & 0.54 & 0.07 & $<.001$ \\
Pre-COVID Diagnosis of MDD & -0.01 & 0.94 & .998 \\
Male Gender & -0.70 & 1.11 & .530 \\
Social Media Exposure * CTQ & 0.03 & 0.01 & .044 \\
Traditional News Media Exposure * CTQ & -0.01 & 0.01 & .568
\end{tabular}

PTSD (PCL-5)

\begin{tabular}{lccc} 
Variable & $b$ & SE & $p$ \\
\hline Time & -0.07 & 0.05 & .162 \\
Social Media Exposure & -4.07 & 1.57 & .011 \\
Traditional News Media Exposure & 2.01 & 1.49 & .178 \\
CTQ & 0.06 & 0.08 & .453 \\
Baseline PTSD (PCL-5) & 0.47 & 0.07 & $<.001$ \\
Pre-COVID Diagnosis of PTSD & 4.70 & 2.49 & .065 \\
Male Gender & -4.02 & 2.85 & .164 \\
Social Media Exposure * CTQ & 0.11 & 0.03 & $<.001$ \\
Traditional News Media Exposure * CTQ & -0.06 & 0.03 & .072 \\
\hline
\end{tabular}

Table 2. Multilevel models with pandemic-related media access predicting depression and PTSD during the first 30 Days of the COVID-19 pandemic.

viewed during the study period had a strong negative valence that in turn amplified the perception of health risk as well as negative affect and ultimately increased symptoms. A recent cross-sectional study indicated that exposure to pandemic-related social media increased negative affect, which in turn increased symptoms of depression and traumatic stress (Zhao Zhou, 2020). Given the strong association between negative affect and childhood maltreatment, those with such histories are posited to be more vulnerable to the onset of negative affect associated with social media exposure (Nanni et al., 2012; Teicher et al., 2006). More nuanced work is needed to evaluate the strength and directionality of these relations to better understand the bidirectional relation between social media exposure and psychopathology.

These findings have implications for clinical work with those with maltreatment histories. Clinicians should assess the amount of social media that clients consume related to the pandemic, particularly when depression or PTSD symptoms are reported. Specific plans and strategies to reduce social media consumption may be useful. Helpful strategies may include turning off notifications on mobile devices to minimize stress-inducing intrusions as well as engaging in safe and pleasurable activities without a mobile device. Planned breaks from COVID-19 related media may be beneficial as well. Furthermore, identifying coping behaviors that do not involve social media may prove beneficial given that those with such symptoms may turn to social media as a coping strategy.

The study had several limitations of note. First, the amount or duration of media consumed per day was not assessed and so it is unclear if more consumption in a given day is associ- 
ated with greater changes in PTSD and depression. Objective data on media consumption should be collected via mobile devices (e.g., ScreenTime) in future work. Second, the sample was majority female and entirely composed of young adults. It is unclear how these findings may generalize to a sample of older adults who may be affected by the pandemic differently. This group may have had concerns about childcare and the presence of chronic health conditions. Relatedly, the sample was majority White and the results may not generalize to individuals of other racial and ethnic groups. Third, the assessment period was at the early phase of the pandemic at the US. More work is needed to determine how these relations changed as the pandemic continued. Fourth, the primary source of COVID-related information - social media, traditional media, or other sources was not evaluated. Participants may have placed different value on information depending on the source, which may in turn have influenced their mental health.

The results from the present study demonstrated that regular social media exposure, or doomscrolling, is associated with an increase in depression and PTSD, even when accounting for pre-pandemic mental health. This effect was magnified for those with histories of maltreatment. This result highlights a major challenge imposed by the COVID-19 pandemic - remaining informed is associated with a mental cost. Strategies to limit doomscrolling and engage in positive activities may offset the detrimental effect of engaging in these behaviors. Exploring the interactive effects of media exposure, related stressors, and protective factors on mental health is imperative to determining the broader impact of the pandemic and how to respond to these events.

\section{References}

Alshaabi, T., Adams, J. L., Arnold, M. V., Minot, J. R., Dewhurst, D. R., Reagan, A. J., Danforth, C. M., and Dodds, P. S. (2021). Storywrangler: A massive exploratorium for sociolinguistic, cultural, socioeconomic, and political timelines using twitter. arXiv:2007.12988 [physics]. arXiv: 2007.12988.

Auxier, B. and Anderson, M. (2021). Social media use in 2021. Technical report. [Online; accessed 2021-04-29].

Bernstein, D. P., Ahuvalia, T., Pogge, D., and Handelsman, L. (1997). Validity of the childhood trauma questionnaire in an adolescent psychiatric population. Journal of the American Academy of Child Adolescent Psychiatry, 36(3):340-348.

Bovin, M. J., Marx, B. P., Weathers, F. W., Gallagher, M. W., Rodriguez, P., Schnurr, P. P., and Keane, T. M. (2016). Psychometric properties of the ptsd checklist for diagnostic and statistical manual of mental disorders-fifth edition (pcl-5) in veterans. Psychological Assessment, 28(11):1379-1391.

Contractor, A. A., Weiss, N. H., and Elhai, J. D. (2019). Examination of the relation between ptsd symptoms, smartphone feature uses, and problematic smartphone use. Social Science Computer Review, 37(3):385-403. publisher: SAGE Publications Inc.

Fullana, M. A., Hidalgo-Mazzei, D., Vieta, E., and Radua, J. (2020). Coping behaviors associated with decreased anxiety and depressive symptoms during the covid-19 pandemic and lockdown. Journal of Affective Disorders, 275:80-81.

Galea, S., Merchant, R. M., and Lurie, N. (2020). The mental health consequences of covid-19 and physical distancing: The need for prevention and early intervention. JAMA Internal Medicine, 180(6):817-818. publisher: American Medical Association.

Gao, J., Zheng, P., Jia, Y., Chen, H., Mao, Y., Chen, S., Wang, Y., Fu, H., and Dai, J. (2020). Mental health problems and social media exposure during covid-19 outbreak. PLOS ONE, 15(4):e0231924. publisher: Public Library of Science.

Gonzales, G., Loret de Mola, E., Gavulic, K. A., McKay, T., and Purcell, C. (2020). Mental health needs among lesbian, gay, bisexual, and transgender college students during the covid-19 pandemic. Journal of Adolescent Health, 67(5):645-648.

Heffner, J., Vives, M.-L., and FeldmanHall, O. (2021). Emotional responses to prosocial messages increase willingness to self-isolate during the covid-19 pandemic. Personality and Individual Differences, 170:110420. 
Holman, E. A., Garfin, D. R., and Silver, R. C. (2014). Media's role in broadcasting acute stress following the boston marathon bombings. Proceedings of the National Academy of Sciences, 111(1):93-98. ISBN: 9781316265116 publisher: National Academy of Sciences section: Social Sciences PMID: 24324161.

Holmes, E. A., O'Connor, R. C., Perry, V. H., Tracey, I., Wessely, S., Arseneault, L., Ballard, C., Christensen, H., Cohen Silver, R., Everall, I., Ford, T., John, A., Kabir, T., King, K., Madan, I., Michie, S., Przybylski, A. K., Shafran, R., Sweeney, A., Worthman, C. M., Yardley, L., Cowan, K., Cope, C., Hotopf, M., and Bullmore, E. (2020). Multidisciplinary research priorities for the covid-19 pandemic: a call for action for mental health science. The Lancet Psychiatry, 7(6):547-560.

Kasperson, R. E., Renn, O., Slovic, P., Brown, H. S., Emel, J., Goble, R., Kasperson, J. X., and Ratick, S. (1988).

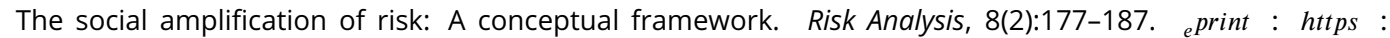
//onlinelibrary.wiley.com/doi/pd f/10.1111/j.1539-6924.1988.tb01168.x.

Kroenke, K., Spitzer, R. L., and Williams, J. B. W. (2001). The phq-9. Journal of General Internal Medicine, 16(9):606613.

Larsen, R. (2011). Missing data imputation versus full information maximum likelihood with second-level dependencies. Structural Equation Modeling: A Multidisciplinary Journal, 18(4):649-662.

Li, Y., Zhao, J., Ma, Z., McReynolds, L. S., Lin, D., Chen, Z., Wang, T., Wang, D., Zhang, Y., Zhang, J., Fan, F., and Liu, X. (2021). Mental health among college students during the covid-19 pandemic in china: A 2-wave longitudinal survey. Journal of Affective Disorders, 281:597-604.

Nanni, V., Uher, R., and Danese, A. (2012). Childhood maltreatment predicts unfavorable course of illness and treatment outcome in depression: A meta-analysis. American Journal of Psychiatry, 169(2):141-151. publisher: American Psychiatric Publishing.

$\mathrm{Ng}$, Y. J., Yang, Z. J., and Vishwanath, A. (2018). To fear or not to fear? applying the social amplification of risk framework on two environmental health risks in singapore. Journal of Risk Research, 21(12):1487-1501. publisher: Routledge ${ }_{e}$ print : https : //doi.org/10.1080/13669877.2017.1313762.

Perlman, M. R., Dawson, A. E., Dardis, C. M., Egan, T., and Anderson, T. (2016). The association between childhood maltreatment and coping strategies: The indirect effect through attachment. The Journal of Genetic Psychology, 177(5):156-171. publisher: Routledge ${ }_{e}$ print : https : //doi.org/10.1080/00221325.2016.1220912.

Schlomer, G. L., Bauman, S., and Card, N. A. (2010). Best practices for missing data management in counseling psychology. Journal of Counseling psychology, 57(1):1.

Sell, T. K., Boddie, C., McGinty, E. E., Pollack, K., Smith, K. C., Burke, T. A., and Rutkow, L. (2017). Media messages and perception of risk for ebola virus infection, united states. Emerging Infectious Diseases, 23(1):108-111. PMID: 27983495 PMCID: PMC5176223.

Sheehan, D. V., Janavs, J., Baker, R., Harnett-Sheehan, K., Knapp, E., Sheehan, M., Lecrubier, Y., Weiller, E., Hergueta, T., Amorim, P., et al. (1998). Mini-mini international neuropsychiatric interview-english version 5.0. 0-dsm-iv. Journal of Clinical psychiatry, 59:34-57.

Teicher, M. H., Samson, J. A., Polcari, A., and McGreenery, C. E. (2006). Sticks, stones, and hurtful words: Relative effects of various forms of childhood maltreatment. American Journal of Psychiatry, 163(6):993-1000. publisher: American Psychiatric Publishing.

Wang, C., Pan, R., Wan, X., Tan, Y., Xu, L., Ho, C. S., and Ho, R. C. (2020). Immediate psychological responses and associated factors during the initial stage of the 2019 coronavirus disease (covid-19) epidemic among the general population in china. International Journal of Environmental Research and Public Health, 17(5):1729. number: 5 publisher: Multidisciplinary Digital Publishing Institute.

Weathers, F. W., Bovin, M. J., Lee, D. J., Sloan, D. M., Schnurr, P. P., Kaloupek, D. G., Keane, T. M., and Marx, B. P. (2018). The clinician-administered ptsd scale for dsm-5 (caps-5): Development and initial psychometric evaluation in military veterans. Psychological Assessment, 30(3):383-395. publisher-place: US publisher: American Psychological Association.

Worsley, J. D., McIntyre, J. C., Bentall, R. P., and Corcoran, R. (2018). Childhood maltreatment and problematic social media use: The role of attachment and depression. Psychiatry Research, 267:88-93.

Yates, T. M., Gregor, M. A., and Haviland, M. G. (2012). Child maltreatment, alexithymia, and problematic internet use in young adulthood. Cyberpsychology, Behavior, and Social Networking, 15(4):219-225. publisher: Mary Ann Liebert, Inc., publishers. 
Zhao, N. and Zhou, G. (2020). Social media use and mental health during the covid-19 pandemic: Moderator role of disaster stressor and mediator role of negative affect. Applied Psychology: Health and Well-Being, 12(4):10191038. ${ }_{e}$ print : https : //iaap - journals.onlinelibrary.wiley.com/doi/pdf/10.1111/aphw.12226. 